\title{
Cis regulatory motifs and antisense transcriptional control in the apicomplexan Theileria parva
}

\author{
Kyle Tretina', Roger Pelle ${ }^{2}$ and Joana C. Silva ${ }^{1 *}$
}

\begin{abstract}
Background: Theileria parva is an intracellular parasite that causes a lymphoproliferative disease in cattle. It does so by inducing cancer-like phenotypes in the host cells it infects, although the molecular and regulatory mechanisms involved remain poorly understood. RNAseq data, and the resulting updated genome annotation now available for this parasite, offer an unprecedented opportunity to characterize the genomic features associated with gene regulation in this species. Our previous analyses revealed a T. parva genome even more gene-dense than previously thought, with many adjacent loci overlapping each other, not only at the level of untranslated sequences (UTRs) but even in coding sequences.

Results: Despite this compactness, Theileria intergenic regions show a pattern of size distribution indicative of monocistronic gene transcription. Three previously described motifs are conserved among Theileria species and highly prevalent in promoter regions near or at the transcription start sites. We found novel motifs at many transcription termination sites, as well as upstream of parasite genes thought to be critical for host transformation. Adjacent genes that could be regulated by antisense transcription from an overlapping transcriptional unit are syntenic between T. parva and P. falciparum at a frequency higher than expected by chance, suggesting the presence of common, and evolutionary old, regulatory mechanisms in the phylum Apicomplexa.

Conclusions: We propose a model of transcription with conserved sense and antisense transcription from a few taxonomically ubiquitous and several species-specific promoter motifs. Interestingly, the gene networks regulated by conserved promoters are themselves, in most cases, not conserved between species or genera.
\end{abstract}

Keywords: Theileria parva, Transcription regulation, Regulatory motifs

\section{Background}

Theileria parva infection induces a lymphoproliferative disease, called East coast fever (ECF), in cattle in eastern, central, and southern Africa, where it causes $>\$ 300 \mathrm{M}$ USD of economic loss per year [1]. T. parva sporozoites in the salivary glands of ticks are introduced into the bloodstream of cattle, where they infect lymphocytes. In the lymphocyte cytoplasm, sporozoites develop into multinucleate schizonts, which transform the infected cell to exhibit several cancer-like phenotypes, including hyperproliferation and replicative immortality [2]. The parasite genes regulating these processes are only beginning to be

\footnotetext{
*Correspondence: jcsilva@som.umaryland.edu

'Institute for Genome Sciences and Department of Microbiology and

Immunology, University of Maryland School of Medicine, Baltimore, MD, USA Full list of author information is available at the end of the article
}

uncovered. However, our understanding of how those genes are regulated remains limited by the lack of knowledge regarding transcriptional regulation in $T$. parva. No genetic modification systems have been developed for $T$. parva, and only recently has a successful transfection system been reported [3], making it exceedingly difficult to investigate gene function and regulation by standard molecular biology approaches in this parasite. Bioinformatics methods provide a powerful complementary approach to molecular biology in the characterization of mechanisms of gene regulation [4].

The identification and characterization of core promoters and transcription start sites is key to understanding gene regulation in any organism [5]. Although early models of RNA polymerase II transcription suggested that a core promoter, such as a TATA box, directs 
transcription from a single defined nucleotide position, recent genome-wide studies favor a more intricate model that includes multiple promoters and start sites for most genes. This is a potentially significant source of transcriptome diversity [6]. Apicomplexans are notable for their lack of canonical eukaryotic transcription factors, and until the relatively recent discovery of the ApiAP2 transcription factor family it was thought that transcription was not a very important mechanism of gene regulation in apicomplexans [7-9]. While Plasmodium species now have extensive transcription factor binding site information for the ApiAP2 family [10], no such resource exists for $T$. parva, even though it could significantly enrich further research and an understanding of its pathogenesis. Interestingly, in Plasmodium, the DNA binding motifs for orthologous ApiAP2 transcription factors are conserved across the genus, but the genes that they regulate are not [10]. It is not, however, known if this is the case within Piroplasmida, the order to which Theileria and Babesia species belong. Here, we use the first published RNAseq dataset for T. parva, as well as a variety of bioinformatics methods to predict cis regulatory motifs in T. parva and other piroplasmids, and investigate the sequence and evolution of genomic motifs that regulate gene expression.

\section{Results and discussion}

Aside from early bioinformatics predictions of potentially functional cis regulatory motifs in the T. parva genome [11], very little is known about the basic transcriptional unit in this species. Those analyses revealed that in $T$. parva $5^{\prime} 5^{\prime}$ intergenic regions (i.e., those flanked by genes in head-to-head orientation) are significantly longer than $5^{\prime}$ 3 ' intergenic regions (genes head-to-tail), which in turn are significantly longer than $3^{\prime} 3^{\prime}$ intergenic regions (genes tailto-tail), a clear signature of spatial requirements imposed by upstream regulatory motifs in a very compact genome [11-13]. A similar pattern is also found in fungal genomes $[14,15]$. Those analyses were based only on a small subset of genes in the original annotation of the T. parva genome $[11,16]$, and without knowledge of the location of transcription start sites. The extensive T. parva RNAseq data recently made available in Genbank formed the basis for the improved re-annotation of its genome (Tretina and Silva, in prep) and provides information on the start and end sites of transcripts and UTR composition in this species. The updated genome annotation, with close to $50 \%$ of the genes with revised coding sequences, together with the RNAseq data form, a powerful dataset to mine for core promoters and other gene transcription regulatory motifs. Even though the motifs identified here are based solely on in silico analyses, we attempt to provide potential biological context and extrapolate on potential implications by drawing parallels with similar observations in other taxa.
Potentially functional cis regulatory motifs include G-box and Spe2 and motifs near transcription termination sites of genes expressed in the $T$. parva schizont stage

We now expand the original $T$. parva analyses to the whole genome, and investigated similar patterns in the other three Theileria species for which a genome assembly is publicly available, namely $T$. annulata, $T$. orientalis and T. equi [16-19]. As would be expected for a genome that includes primarily monocistronic transcriptional units, the ratio of $5^{\prime} 5^{\prime}: 5^{\prime} 3^{\prime}: 3^{\prime} 3^{\prime}$ intergenic regions is approximately 1:2:1, respectively (Table 1 ). The rather small size of the intergenic regions, and the correlation between their length and the number of flanking $5^{\prime}$ gene ends, indicate that genome space is tightly regulated and at a premium in these organisms, with longer intergenic regions reflecting increased functionality added by cisacting elements (Table 1, Fig. 1).

We then searched for additional cis-regulatory motifs in the T. parva genome. The extremely biased content in intergenic regions in Plasmodium, with GC nucleotide content close to $10 \%$, hinders the application of some motif-finding tools [20]. Theileria species do not have the same limitation, since they have considerably smaller intergenic regions, with higher GC content. De novo motif searches in Toxoplasma gondii, which has a relatively GC-rich genome, identified potential cis regulatory motifs that are different from any that have been identified in T. parva [21]. Here, specific genomic regions were extracted and motifs found to be enriched in these sequences were identified de novo using the MEME suite. The distribution of these motifs relative to transcription or translation initiation or termination sites was determined with FIMO, and their distribution in different genomic regions was determined with MAST (Methods).

\section{Upstream motifs}

Recent work in the piroplasma species Babesia bovis has identified the consensus sequence TYAYWWW with a tight distribution around transcription start sites, that suggests similarity to an initiator-like motif [22]. As validation to our updated gene models, we have also found this motif with start site peaking in distribution 3 bp upstream of transcription start sites in the T. parva genome (Fig. 2).

Since the typical size of promoter regions in T. parva is unknown, MEME searches were conducted on genomic sequences of 50, 100, and 150 base pairs centered on the annotated transcription start site of all mRNAs that do not overlap other transcripts. Many identical motifs were identified in all three searches, with three distinct motifs found among the top four (Additional file 1: Figure S1), suggesting a tight distribution around the transcription initiation sites. Three of these motifs were previously 
Table 1 Comparisons of the number, type, and size of intergenic regions (IGR) in four sequenced Theileria parasites (T. parva, T. annulata, T.orientalis, and T. equi)

\begin{tabular}{|c|c|c|c|c|c|}
\hline Species & IGR Type ${ }^{b}$ & $n$ & Ratio of IGR types ${ }^{b}$ & Median Size & Ratio of Median Size ${ }^{b}$ \\
\hline \multirow[t]{3}{*}{ T. parva } & $5^{\prime} 5^{\prime}$ & 851 & 1.00 & 493 & 3.21 \\
\hline & $5^{\prime} 3^{\prime}$ & 1968 & 2.30 & 309 & 2.01 \\
\hline & $3^{\prime} 3^{\prime}$ & 854 & 1.00 & 154 & 1.00 \\
\hline \multirow[t]{3}{*}{ T. annulata } & $5^{\prime} 5^{\prime}$ & 908 & 1.00 & 438 & 3.49 \\
\hline & $5^{\prime} 3^{\prime}$ & 1949 & 2.14 & 277 & 2.21 \\
\hline & $3^{\prime} 3^{\prime}$ & 910 & 1.00 & 126 & 1.00 \\
\hline \multirow[t]{3}{*}{ T. orientalis } & $5^{\prime} 5^{\prime}$ & 1056 & 1.00 & 424 & 2.09 \\
\hline & $5^{\prime} 3^{\prime}$ & 1838 & 1.74 & 302 & 1.49 \\
\hline & $3^{\prime} 3^{\prime}$ & 1054 & 1.00 & 203 & 1.00 \\
\hline \multirow[t]{3}{*}{ T. equi } & $5^{\prime} 5^{\prime}$ & 1203 & 1.00 & 645 & 4.76 \\
\hline & $5^{\prime} 3^{\prime}$ & 2853 & 2.38 & 394 & 2.91 \\
\hline & $3^{\prime} 3^{\prime}$ & 1198 & 1.00 & 136 & 1.00 \\
\hline
\end{tabular}

IRG type: genes in head-to-head (5'5'), head-to-tail (5'3') and tail-to-tail (3'3') orientation. ' $R$ Ratio used as a reference (denominator) the value for 3'3' IGRs

identified [11], and our expanded analyses find additional information on their localization: in particular, two of the motifs were found predominantly near or at annotated transcript start sites, and were located most often in $5^{\prime} 5^{\prime}$ intergenic regions compared to other intergenic regions, coding regions, UTRs or introns, a pattern that holds true in all four Theileria species with an annotated genome assembly (Fig. 3, Additional file 2: Table S1).

One of these motifs, CCCCAT, shares a high level of similarity to the G-box motif found to be functional upstream of heat-shock proteins in Plasmodium species. This motif is bound by the P. falciparum ApiAP2
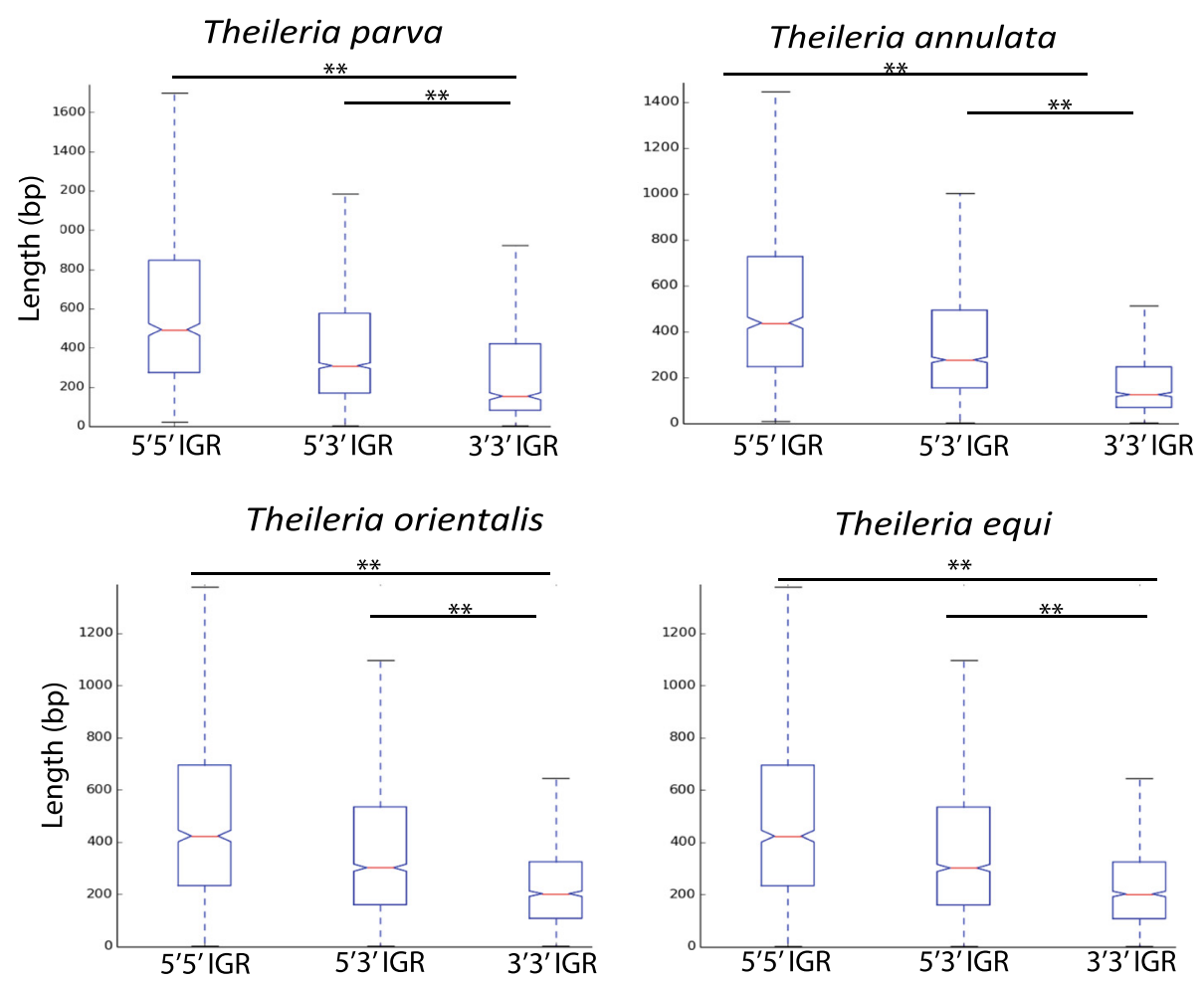

Fig. 1 Length of the three types of intergenic region (IGR) length in the genomes of T. parva, T. annulata, T. orientalis, and T. equi. IGR length, shown in base pairs, correlates with the directionality of the transcriptional units that flank them. Boxplot shows median (orange line), second and third quartiles (within box) and max value below $75 \%+1.5$ interquartile range and minimum value above $25 \%-1.5$ interquartile range (whiskers). Comparisons of intergenic region types within each species were significantly different than each other $(0.001<p<0.005$, two-tailed Student's $t$-test) 


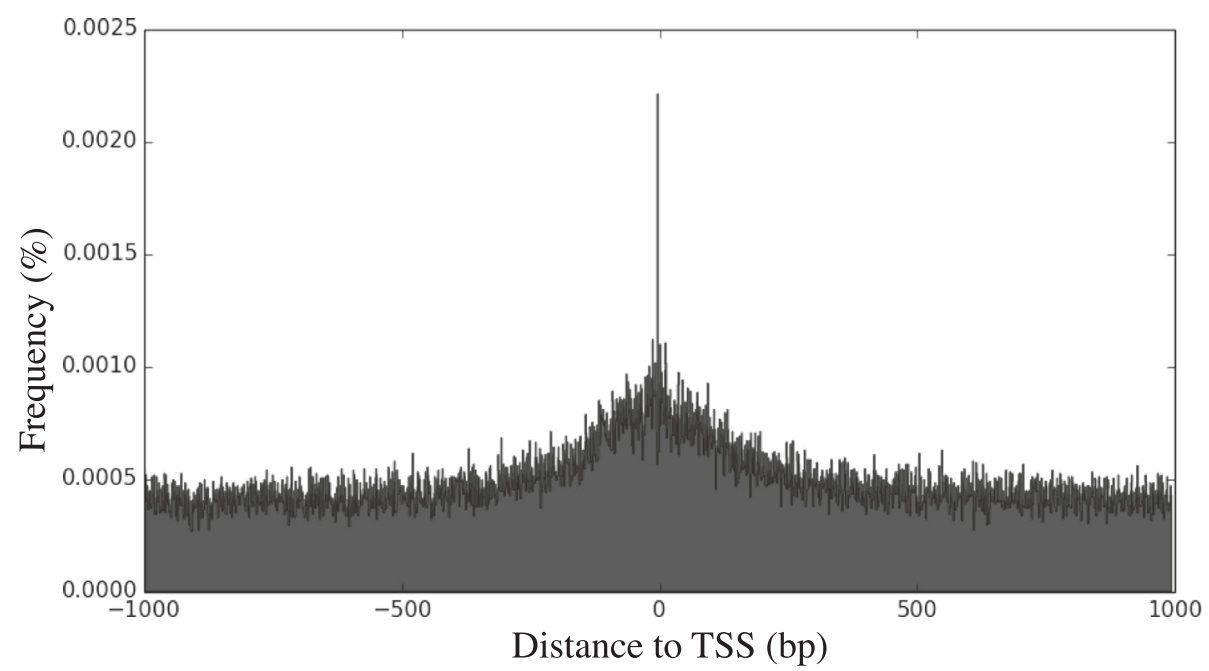

Fig. 2 Distribution of the TYAYUWW motif around transcription initiation sites. The horizontal axis represents sequence areas from $-1,000 \mathrm{bp}$ to $+1,000 \mathrm{bp}$ around transcription initiation sites with single nucleotide resolution. Position 0 represents the transcription initiation site, with a peak observed at the -3 position. The vertical axis represents the normalized frequency of the motif

transcription factor PF13_0235_D1 [10], which does not have a bioinformatically identifiable homolog in $T$. parva. PF13_0235_D1 was found to be upstream of ribosomal and proteins involved in the unfolded protein response, indicating that this transcription factor regulates a biologically critical gene network. This motif has a very localized distribution in $B$. bovis, where it peaks 50 bp upstream of transcriptional start sites and has been implicated as an essential part of the core promoter [22]. A protein in C. parvum (cgd8_810) binds this motif, but the domain ortholog in T annulata (TA12015) does not [23]. A divergence in the function of the gene network regulated by the G-box motif in T. annulata is also indicated by the finding that it is enriched in promoters of genes that are up-regulated from the merozoite to piroplasm stages [23].

The second motif, AATGTGTAA, consists of a completely conserved core of five thymine and guanine nucleotides, with the two flanking and more labile adenines at each end. The core is identical to a motif over-represented in B. bovis promoter regions [22]. Interestingly, the orthologs TA11145 of T. annulata and PF3D7_0802100 (formerly MAL8P1.153) of P. falciparum, both have been shown to bind this motif [23]. In fact, a family of transcription factors conserved throughout the haematosporidians have been predicted to be auto-regulated and compete for binding to these motifs as a critical step in the stochastic differentiation process from macroschizont to merozoite life cycle stages [23]. A third motif, GATTCCA, is similar to the motif bound by NFkB in mammals [24]. Previous work has established this motif as being enriched upstream of genes involved in protein fate (i.e. post-translational modification, degradation/ stabilization, and targeting), and a BLASTP analysis identified a $T$. parva protein, TP02_0125, to have and domain with $55 \%$ identity to the DNA-binding domain of NFkB and therefore predicted to bind this motif [11]. Our BLASTP searches also identify strong homology ( $>80 \%$ identity over $>70 \%$ of the gene) to proteins in $T$. annulata (TA11490), T. orientalis (TOT_020000113), and B. bovis (BBOV_IIII010230), but not in T. equi, where the best hit (BEWA_051930) had only $41 \%$ identity over $13 \%$ of the gene. Interestingly, and possibly not coincidentally, this third motif was not found in T. equi.

\section{Downstream motifs}

The region downstream of genes may contain motifs involved in both termination of sense transcripts and the initiation of antisense transcripts encoded in the opposite strand. Antisense transcripts can arise from promoters near the terminal ends of their sense counterparts, and their expression is regulated by many of the same factors as sense genes [25]. Eukaryotic transcription termination is typically assumed to use the same mechanisms as model yeast systems, where the RNA polymerase II transcribes well past the polyadenylation signal (PAS), but a series of allosteric protein-protein interactions result in the cleavage of the transcript 11-30 bp downstream of the PAS and the degradation of the remaining RNA by endonucleases $[26,27]$. Since there are no cis regulatory motifs reported to be involved in antisense transcription or transcription termination in T. parva, we searched for motifs around the annotated 3' ends of transcripts. To this end, MEME searches similar to those described for promoter regions were conducted around transcript termination sites of annotated mRNAs. A few motifs were found to be over- 


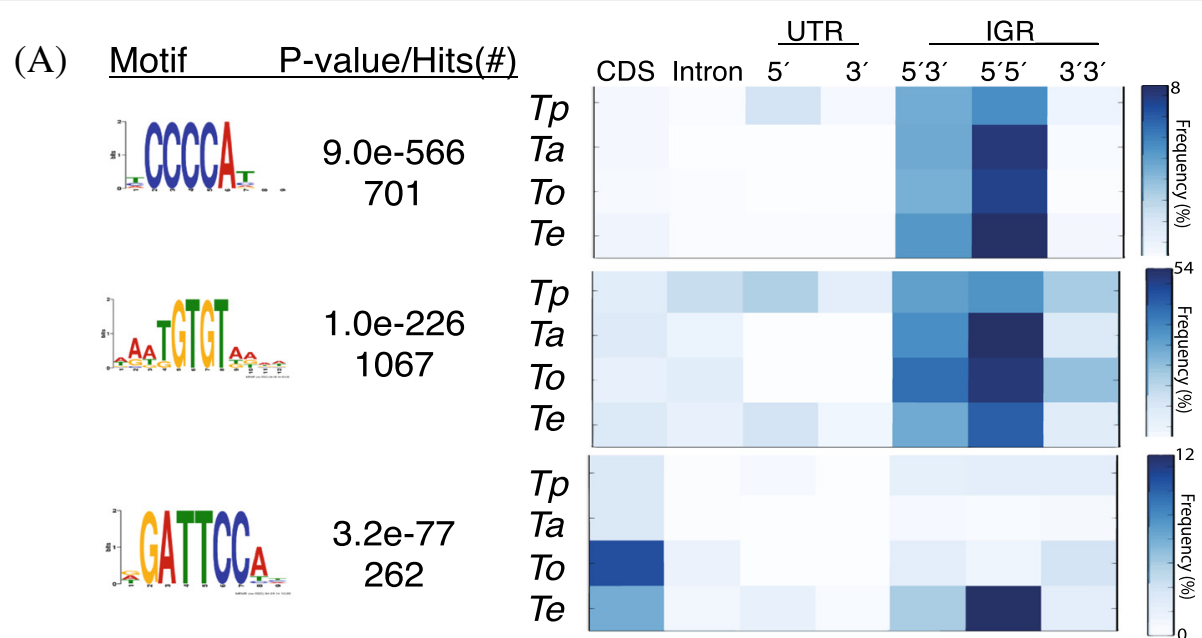

(B)

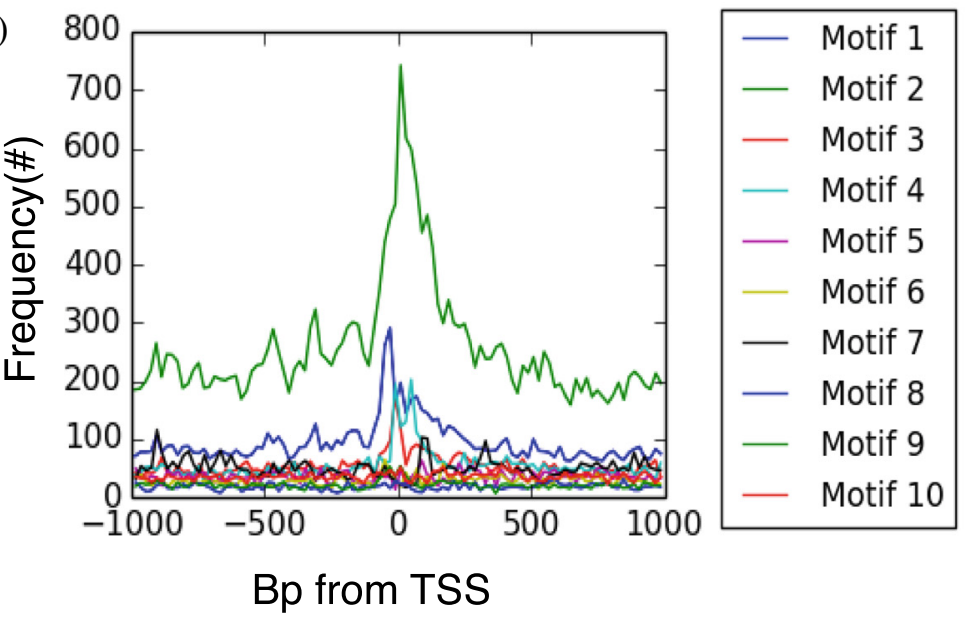

Fig. 3 Metrics of the top 3 motifs identified by MEME searches around transcription initiation sites. a The top three motifs (G-box, Spe2, and NFkB-like), their p-value identified by MEME, and their frequency in different genomic regions of four Theileria species and in the three types of intergenic regions (IGR) (Tp = Theileria parva; Ta = Theileria annulata; To = Theileria orientalis; $T e=$ Theileria equi). $\mathbf{b}$ Distribution of the three motifs in the vicinity of transcription initiation sites in T. parva. All three motifs are located predominantly at or near transcription start sites (TSS)

represented around the 3' end of transcripts, some of which were AT-rich and/or conducive to forming hairpinlike structures (Additional file 1: Figure S2c). One of these motifs (Additional file 1: Figure S2a, 50 bp region, motif 4) was identified in all $3^{\prime}$ motif searches, was enriched in 3'3' intergenic regions of all four sequenced Theileria species (Fig. 4a, Additional file 2: Table S1), and was absent around transcription initiation sites. This AT-rich motif had pronounced peaks in distribution at transcript 3 ' ends (Fig. 4b). Given the high AT-content and selfcomplementary nature of these motifs, with their hairpin potential, it could also be postulated that they may play a structural role in transcription termination. Interestingly, another of the motifs (Additional file 1: Figure S2a, 25 and 50 bp regions, motif 1 ) is identical to the central region of the second upstream cis-regulatory motifs described above. Therefore, it is tempting to postulate a possible double role for this motif in both transcription termination and antisense transcription initiation.

3' UTRs also can play an additional significant role in gene regulation $[28,29]$ by potentially containing a signal for polyadenylation. Since the RNAseq protocol used in the present study included the enrichment of polyadenylated transcripts, we then took advantage of this dataset in order to predict possible canonical and noncanonical polyadenylation signals (PAS). It should be noted that non-polyadenylated, high-AT content parasite transcripts might also be pulled down using this protocol. With a previously established algorithm [30], we discovered that $T$. parva most likely uses a PAS that closely resembles, but is not identical to, that of other eukaryotes, including mammals, and found several other hexamers that we predict to play a role in polyadenylation (Additional file 2: Table S2). 
(A)

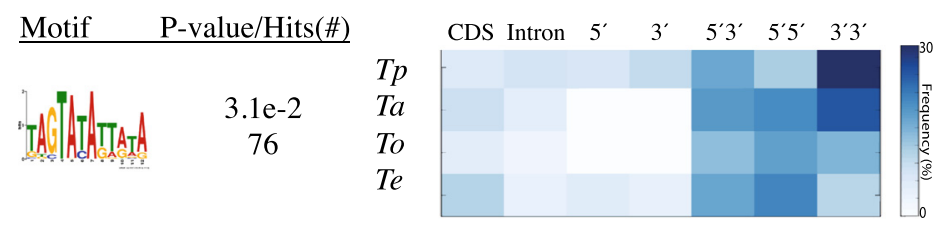

(B)

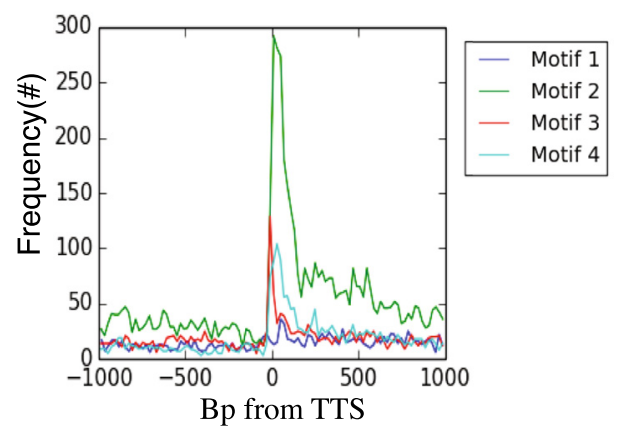

Fig. 4 Metrics of the top motif identified by MEME searches of transcription termination sites. a Motif 2 was conserved in tail-to-tail intergenic regions in four Theileria species ( $T p=$ Theileria parva; $T a=$ Theileria annulata; $T o=$ Theileria orientalis; $T e=$ Theileria equi) and $\mathbf{b}$ peaked in distribution at transcription termination sites genome-wide in T. parva. The horizontal axis represents sequence areas from -1,000 bp upstream to $+1,000$ bp downstream around transcription initiation sites with single nucleotide resolution. Position 0 represents the transcription termination site. The vertical axis represents the frequency of the motif

\section{UTR motifs and non-coding exons}

Motifs found in 5' UTRs contribute to post-transcriptional gene regulation in a number of organisms [31]. In $T$. parva, annotated 5' UTRs, with median size of close to $150 \mathrm{bp}$, are significantly longer than 3' UTRs, of median length $<50 \mathrm{bp}$ (Additional file 1: Figure S3), a pattern also found in Plasmodium and Babesia [13, 22]. Together with the presence of $>100$ RNA binding proteins in $T$. parva (Additional file 2: Table S3), this pattern is highly suggestive of a functional role for $5^{\prime}$ UTRs. In order to identify potential regulatory motifs encoded in UTRs, four separate motif searches were conducted: searches in $5^{\prime}$ and $3^{\prime}$ UTRs longer than $8 \mathrm{bp}$, each separated into those that either did or did not overlap an adjacent gene model. Out of these four UTR sets, conserved motifs were only identified in 5' UTRs that do not overlap other transcripts (Additional file 1: Figure S4a, all motifs summarized in Additional file 1: Figure S4b). The motifs identified in these "non-overlapping" 5' UTRs are considerably longer than the potential regulatory motifs found near transcription start sites and are rich in $G$ and $\mathrm{C}$ nucleotides (GC-rich). The GC content of UTRs can affect protein translation efficiency; elevated GC content is associated with a decrease in translation efficiency [32]. The fact that the motifs found in these regions are $\mathrm{GC}$-rich may indicate their role in posttranscriptional regulation.

Another mechanism by which 5' UTRs have been found to impact gene expression is through the synthesis of small, upstream open reading frames (uORFs). In Arabidopsis, uORFs are present upstream of genes that encode regulatory products such as transcription factors [33]. Recent evidence suggests that they might play a role in translation delay in $P$. falciparum, perhaps by delaying translation of the main ORF or by encoding regulatory peptides themselves [34]. Small peptides translated from uORFs could also possibly be presented antigens on host MHC I, just like host defective ribosomal products [35], making uORFs important features to report. At least $5 \%$ of $T$. parva genes have potential uORFs; 217 genes have at least one uORF that starts with a methionine, often considered the minimal ORF length for bona fide protein-coding gene, indicating that these peptides might play a significant role in T. parva biology (Fig. 5).

\section{Antisense transcription is highly prevalent in the T. parva genome and constitutes a potentially conserved mechanism of gene regulation}

Antisense transcripts can play a role in the cis regulation of sense gene expression via epigenetic, transcriptional, post-transcriptional, or even translational interference mechanisms. They can also play trans regulatory roles in the expression of other genes in the genome [25]. Overlapping transcripts encoded on opposite strands, which are widespread in the T. parva genome and can result from run-through transcription (Tretina and Silva, in prep), have the potential to affect the expression level of neighboring gene when RNA polymerases transcribing in opposite directions collide [36, 37]. It is important to note that the temporal expression of overlapping genes cannot be determined from the RNAseq data used in the 


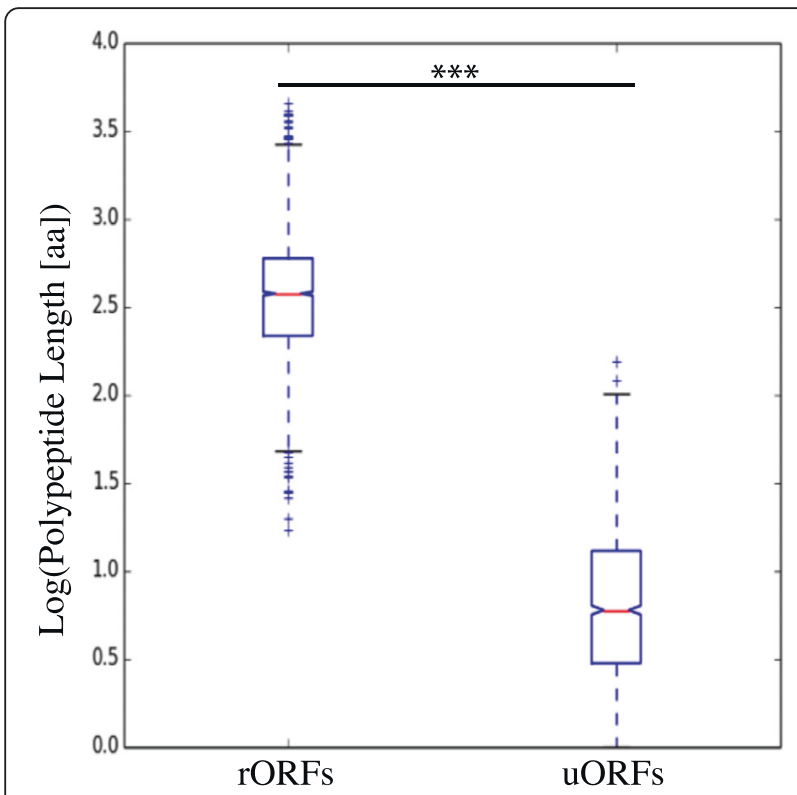

Fig. 5 Length distribution of upstream open reading frames (uORFs) and reference open reading frames (rORFs). Only ORFs starting with a methionine were included in these distributions. Legend as in Fig. 1, with outliers are represented by ' + '. Boxplot shows median (orange line), second and third quartiles (within box) and max value below $75 \%+1.5$ interquartile range and minimum value above $25 \%-1.5$ interquartile range (whiskers). uORF lengths were significantly lower than than rORF lengths ( $p<0.001$, two-tailed Student's $t$-test)

present study, since the RNA was extracted from unsynchronized cells.

Given the large percentage of overlapping transcripts from opposite strands in this genome (Tretina and Silva, in prep), the question arises of whether overlapping antisense transcription is merely "leakage" of the transcription machinery in a highly compact genome, or if instead it is the reflection of a carefully orchestrated transcriptional process, molded by selective pressure to optimize the level and timing of gene expression. Previous studies in humans have taken a comparative genomics approach to this question, assuming that if consecutive pairs of genes encoded on opposite strands improve organismal fitness (e.g., by temporal expression interference) then there will be strong selective pressure to preserve their synteny. In fact, they found that $23.3 \%$ of human adjacent gene pairs encoded in opposite strands (i.e., those in tailto-tail or head-to-head orientation), with overlapping transcripts, have conserved synteny in the pufferfish Takifugu rubripes, compared to only $13.5 \%$ of consecutive head-to-tail gene pairs, i.e., those encoded in the same DNA strand [38]. We find similar evidence in T. parva: while of the 805 head-to-tail consecutive gene pairs in $T$. parva Muguga with orthologs in P. falciparum only $16 \%$ have the same syntenic position in the genome of the latter species, the proportion is larger for pairs of consecutive genes encoded in opposite strands (23 \%) and more than doubles to $35 \%$ for genes pairs with evidence of potential transcriptional interference (Table 2). Therefore, we hypothesize that antisense overlapping of transcripts constitutes an important mechanism of transcriptional regulation in $T$. parva. Antisense transcripts are increasingly recognized as a vital part of gene regulation and antigenic variation in Plasmodium [39], particularly in the regulation of expression of virulence genes [40]. Given the paucity of identifiable transcription factors in these parasites [7, 41], antisense transcription may play a much more prominent role in gene regulation in apicomplexans than previously appreciated.

\section{Unique cis regulatory motifs regulate multi-gene families that are potentially involved in host pathogenesis and parasite immune evasion}

Many apicomplexans have lineage-specific, telomeric multigene families which are often involved in hostpathogen interactions and evasion of the host immune system [42]. In T. parva, the Subtelomere-encoded Variable Secreted Protein (SVSP) family is the largest gene family and has been suggested to play a role in either immune evasion or the manipulation of host cell gene expression $[2,16,43]$. Another gene family, the $T$. parva Host Nucleus (TpHN) family, is homolous to a family of genes in $T$. annulata which have been shown to localize to the host nucleus and alter host gene expression [17, 44]. Given the interest in these gene families as potentially significant in the pathogenesis of ECF, we ran motif searches upstream of all genes in each of the top 20 gene families. Since little is known about

Table 2 Conservation of orientation of homologous gene pairs between Theileria parva Muguga and Plasmodium falciparum 3D7

\begin{tabular}{llll}
\hline $\begin{array}{l}\text { Classification of Theileria } \\
\text { gene pairs }^{\mathrm{a}}\end{array}$ & $\begin{array}{l}\text { Theileria consecutive gene pairs with orthologs in } \\
\text { P. falciparum }\end{array}$ & $\begin{array}{l}\text { Pairs in Theileria with conserved synteny in } \\
\text { Plasmodium }^{\mathrm{b}}\end{array}$ & $\begin{array}{l}\text { Percent conserved } \\
\text { pairs }^{\mathrm{d}}\end{array}$ \\
\hline Opposite strand & 940 & 64 & 22.66 \\
Opposite strand Overlap & 182 & 64 & 35.16 \\
Same-strand & 805 & 125 & 15.53 \\
\hline
\end{tabular}

${ }^{a}$ Classification of groups of Theileria consecutive gene pairs. Opposite strands: pairs of genes in head-to-head or tail-to-tail orientation, according to the $2014 T$. parva Muguga annotation. Antisense: gene pairs for which a sesne-antisense relationship was observed in RNAseq data. Same strand: pairs of consecutive genes that are encoded on the same strand (head-to-tail orientation). ${ }^{\mathrm{b}}$ Pairs of consecutive orthologous genes that preserved their gene order and orientation in T. parva Muguga and P. falciparum 3D7. 'Pairs of genes that are consecutive on the $T$. parva Muguga genome and have orthologs in the $P$. falciparum 3D7 genome. ${ }^{d}$ Percentage of linked pairs 
the regulation of their expression, predictions of cis regulatory motifs that control the expression of these genes can provide rich information for future investigations. MEME searches of potential promoter regions of these genes revealed novel motifs that vary significantly from the motifs found in the previously mentioned motif searches (Additional file 2: Tables S4-S19). For the SVSP and TpHN gene families, several of these motifs showed conserved distribution relative to transcription initiation sites (Additional file 2: Table S20) as well as conserved enrichment in $5^{\prime} 5^{\prime}$ intergenic regions (Additional file 2: Tables S21-S25), indicating that they are likely functional

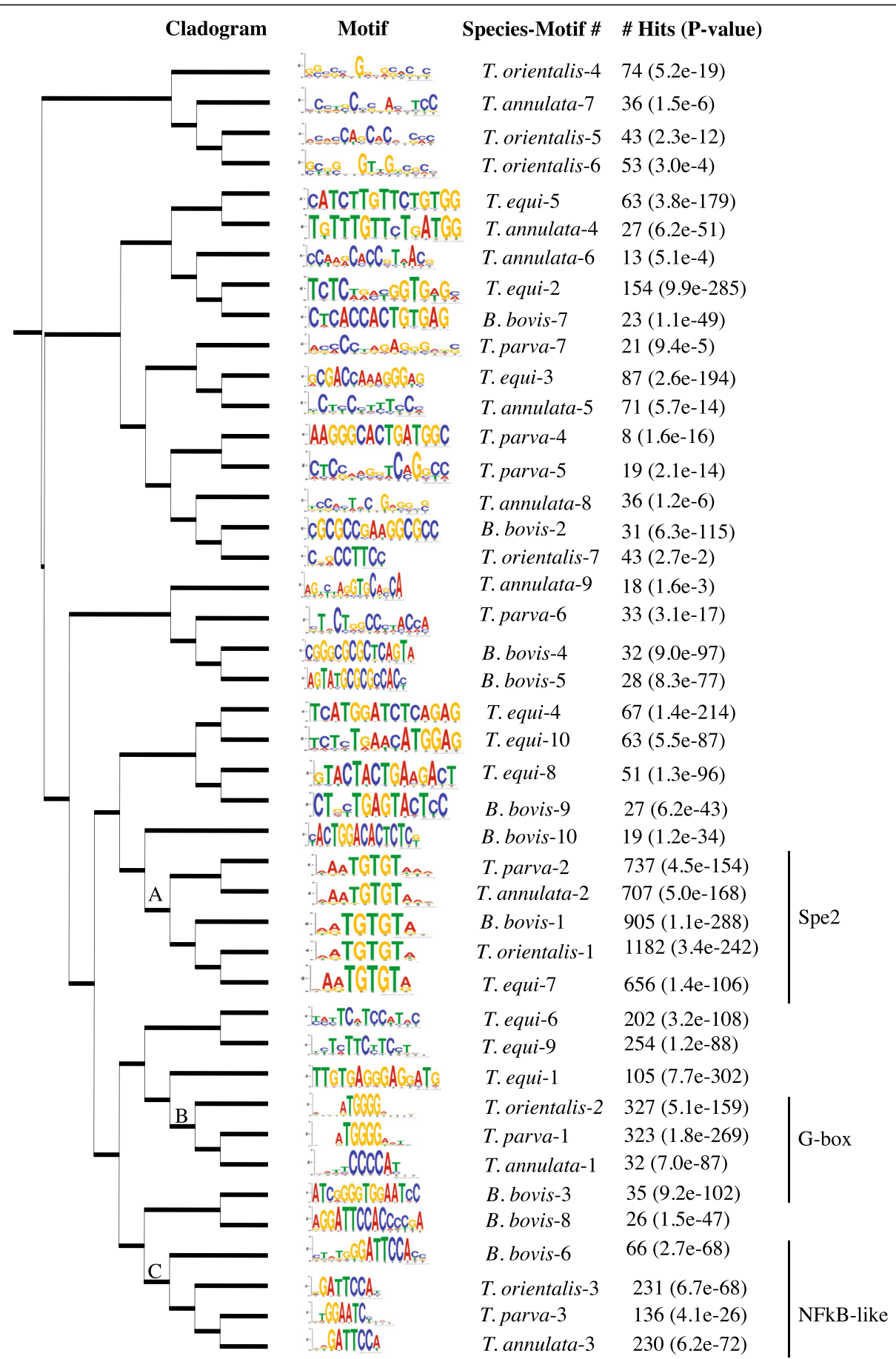

Fig. 6 The relationships of motifs found in T. annulata, T. equi, T. orientalis, T. parva, and B. bovis intergenic regions. The tree was created with STAMP and the tree generated with iTOL (Methods). $A=$ Spe2 motif; B = G-box motif; C = NFkB-like motif 


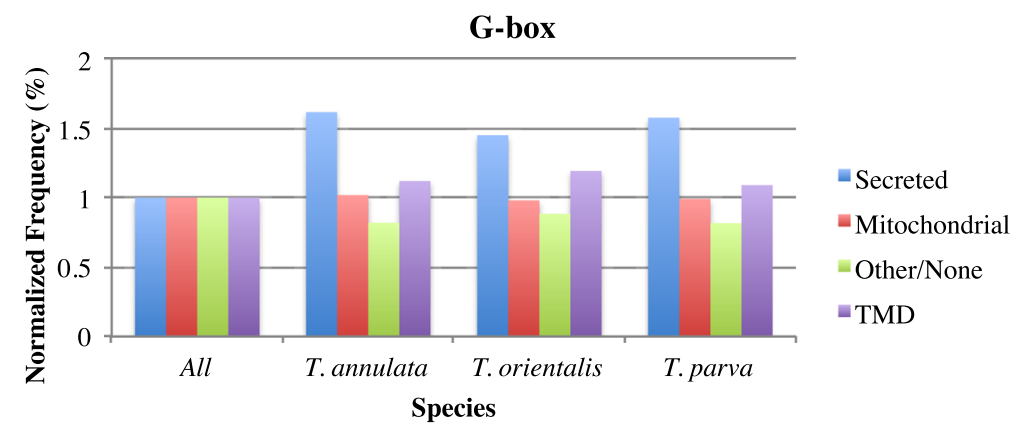

Fig. 7 Theileria genes regulated by the G-box motif are enriched in genes that encoded secreted or transmembrane proteins

and specific to the regulation of these genes, possibly acting in concert with the most common T. parva upstream motifs mentioned above, which are also found associated with many genes in these families.

Gene regulatory motifs and their corresponding networks are not well conserved within piroplasmids

A set of regulatory motifs and the genes they regulate can be maintained through evolutionary time, or they may evolve in one of several ways: 1) a motif sequence may remain conserved, but the genes it regulates vary through time; 2) the motif sequence may evolve while the genes it regulates remain constant; 3 ) there is both a change in (or deletion of) the motif sequence and a rewiring of the networks of co-regulated genes. DNA binding motifs for orthologous ApiAP2 transcription factors are conserved between $P$. falciparum and Plasmodium vivax, although the genes that they regulate are not [10], reminiscent of scenario (1) above. This conservation occurs despite the fact that the two species are distantly related and likely diverged from each other over 60 million years ago [45].

In order to assess the conservation of the primary upstream cis regulatory motifs identified in the piroplasmids, we extracted all intergenic regions 8-300 bp long from four Theileria and one Babesia species, B. bovis, and searched for over-represented motifs and their conservation across species. In contrast to the conservation of possibly multiple ApiAP2 binding motifs across Plasmodium species, among the most common motifs found in Theileria species, only three are shared by two or more species (Fig. 6). These are the same three most conserved and significant motifs in $T$. parva, including the G-box and the Spe 2 motifs. In addition, we find that these motifs do not regulate the same gene networks (Additional file 2: Table S26), suggesting a motif turnover pattern similar to that found in other eukaryotes [46]. Out of these three motifs, we found that the G-box motif is enriched upstream of secreted or transmembrane protein-encoding genes in T. annulata and $T$. orientalis, as well as in T. parva [11], although this motif is not found among the top 10 motifs in T. equi (Fig. 7). Since secreted and transmembrane pathogen proteins are often immunogenic, the association with the G-box motif may have important implications for the study of interactions between Theileria species and their hosts. Finally, the NFkB-like motif was found upstream of transmembrane motifs in B. bovis, but not in Theileria species, which may indicate a distinct divergence in function between these piroplasmid gene networks (Fig. 8). The fact that only two motifs are common to all Theileria species, and that the sets of genes downstream

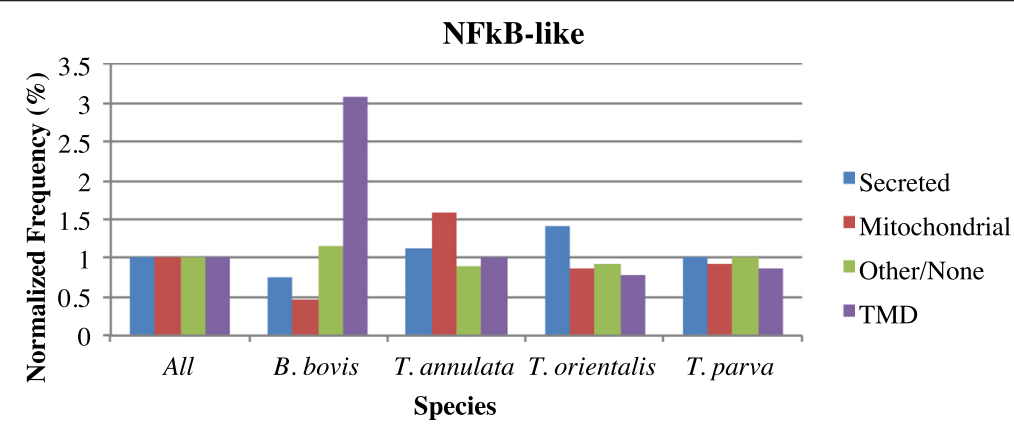

Fig. 8 B. bovis genes regulated by the NFkB-like motif are enriched in genes that encoded transmembrane proteins 
of each motif differs between species, indicates that in fact both motifs and gene networks evolve at a significant rate in this genus, suggestive of scenario (3).

In order to further investigate the rate of motif turnover in these piroplasms, we looked at the G-box, Spe2 and NFkB-like motifs as prototypical piroplasm upstream regulatory motifs. For each set of orthologous genes present in the four Theileria species for which at least one is downstream of one of these motifs, we mapped the number of times each motif was gained or lost (Fig. 9). Despite the fact that we only have available a limited number of taxa for this analysis, a few patterns are clear. First, motif gain is much more prevalent than motif loss, and that in most instances the presence of a motif upstream of a specific gene is a species-specific occurrence. Also, T. parva appears more prone to motif loss than T. annulata (defined by cases in which $T$. orientalis and $T$. annulata, but not $T$. parva, share a motif upstream of an orthologous gene). Finally, the
Spe2 motif seems to be more labile than the other two, with a hundreds of instances of gains and possibly losses (Fig. 9).

Our analyses show that, overall, motifs are most conserved among the most closely related species, $T$. parva and $T$. annulata, and that their sequence conservation decreases with taxonomic distance. T. equi is evolutionarily quite distant from the other Theileria species and exhibits fundamental biological differences [18], and it is perhaps not surprising that is differs in its complement of regulatory motifs. The difference between the pattern seen among species in the genus Plasmodium and those in the order Piroplasma could be a reflection of either the older age of the most recent common ancestor of the Piroplasma taxa, a more conserved functional homology genome-wide within the genus Plasmodium than across the piroplasmids, or a combination of both. A potential caveat to this conclusion is the possibility that the annotation of some of the piroplasmid

(A)

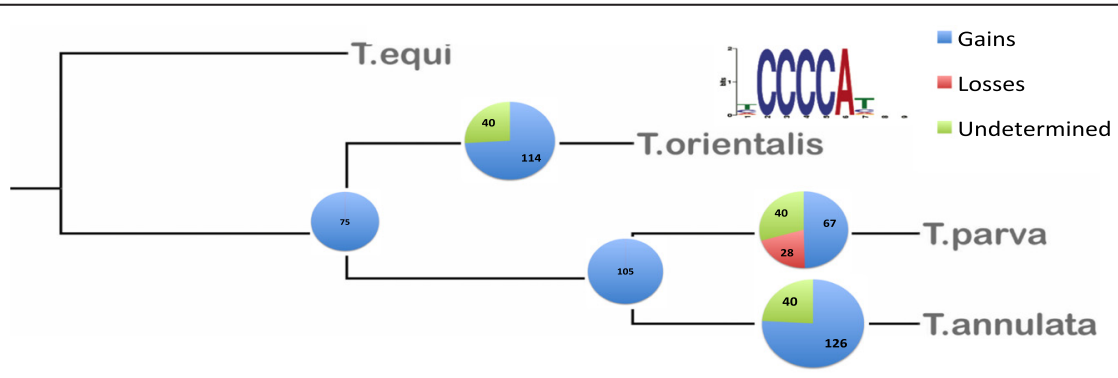

(B)

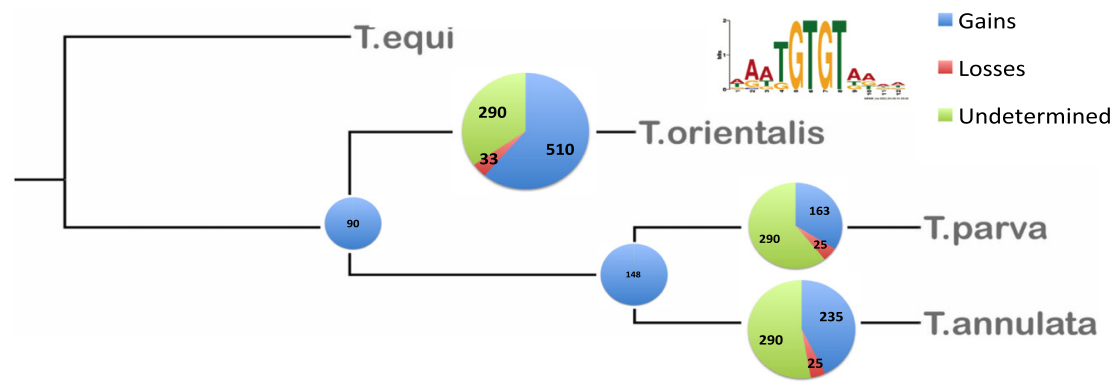

(C)

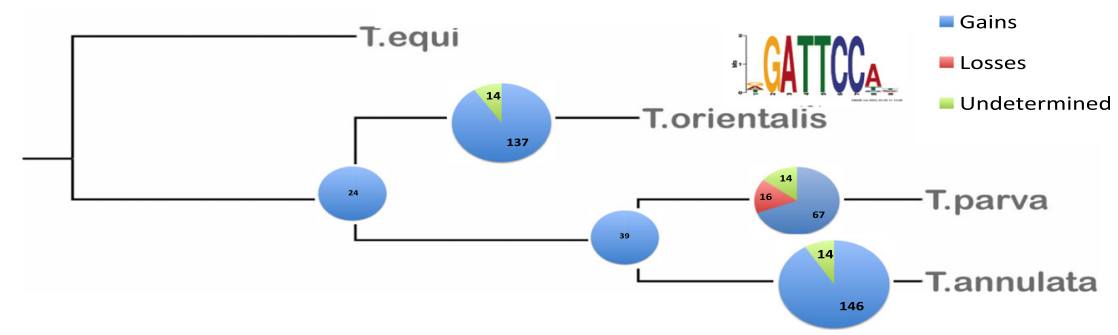

Fig. 9 Reconstruction of turnover rate of three motifs from the present study. a G-box motif; b Spe2 motif; c NFkB-like motif. For each occurrence of a motif upstream of a gene in T. parva (Tp), T. annulata (Ta), T. orientalis (To) and T. equi (Te), we determined its distribution upstream of the ortologs in the other species, and infer the most parsimonious scenario for the number of acquisitions and losses of the motif in the branches leading to To, Ta and Tp. For example, a motif present upstream of the same ortholgous gene in To, Ta and Tp but absent in Te, is inferred to have arisen in the lineage leading to all the first three species, and hence be present at the node reflecting their most recent common ancestor. Motifs present in Te and one of the other species could have been lost twice, or lost once and regained, and so are deamed "undetermined" 
genomes contain significant errors, which prevent the identification of promoter sequences. As the quality of genome annotations improve, and more Theileria genomes become available, an analysis with additional granularity will facilitate a more accurate quantification of motif turnover.

\section{Conclusions}

The highly compact nature of the Theileria genome seems to have resulted in complex regulation of gene transcription, such as the apparently critical role of antisense transcription of adjacent, overlapping genes. While there is an absence of canonical eukaryotic promoter elements, there do seem to be a small set of conserved promoter sequences that regulate large gene networks. While those networks differ among Theileria species, the G-box motif appears to be critical to the regulation of genes involved in host-pathogen interactions.

\section{Methods}

T. parva RNA was isolated from a culture of an available infected bovine lymphocyte line (Tretina and Silva, in prep). No live animals were used in this research. This work required no ethical approval.

The analyses relied on the genome assembly and annotation of $T$. annulata, $B$. bovis, $T$. equi, $T$. orientalis available through PiroplasmaDB (piroplasmadb.org) release 23. For T. parva, we used the extensively updated genome annotation, described elsewhere. In short, a wide array of lines of evidence were used to improve the annotation of the most prevalent isoform for each $T$. parva gene. The sources of evidence include RNAseq read alignments and transcript assemblies, trainable and $d e$ novo gene prediction programs, and protein alignments and expressed sequence tags from $T$. annulata and other apicomplexans. 5' untranslated regions (UTRs) were defined as the genomic sequence between the annotated transcription start sites (TSS) and the translation initiation codon, and 3' UTRs were similarly defined as the genomic sequence between the translation termination codon and the annotated transcription termination site (TTS). Transcription initiation and terminations sites were defined by the presence of an uninterrupted set of reads mapped to the genome, which has a defined start and end (i.e., this set of reads does not overlap a flanking transcriptional unit encoded in the same strand). An upstream open reading frame (uORF) was defined as any open reading frame in the $5^{\prime}$ UTR that starts with a methionine, but that is out of frame with the primary product encoded by the locus.

Searches with the MEME program [47] used motif length constraints of $5 \mathrm{bp}$ to $15 \mathrm{bp}$, searching both strands at the DNA level for a maximum of 10 motifs with an E-value cutoff of 0.05 assuming zero or one occurrence of a motif as has been done previously [11].
Motif search regions are indicated in the text. All searches with FIMO [48] shown had a p-value cutoff of 0.0001. MAST [47] searches were run with the "-hit_list" option and an e-value cutoff of 100. Meme motif positionspecific scoring matrices were aligned in STAMP to identify motif similarity to newly discovered and known motifs [49]. Motifs were searched against a collection of databases including two comprehensive eukaryotic motif databases JASPAR [50] and TRANSFAC [51], FLY [52], FlyReg [53] and Agris [54], AthaMap [55], PLACE [56], Uniprobe [57], other yeast motifs $[58,59]$, as well as the prokaryotic motif databases RegTransBase [60] and DPInteract [61]. The Smith-Waterman local alignment method with a similarity score defined by Pearson's correlation coefficient estimated motif similarity. The STAMP motif phylogenetic tree was generated in iTOL [62].

Orthologous clusters (OCs) were created using the predicted peptide sequences for T. annulata, T. equi, $T$. orientalis, $T$. parva and B. bovis. Jaccard-filtered OC analysis and multigene family clustering were used to construct the final ortholog set as described previously [16]. In brief, an all-versus-all BLASTP was performed, followed by orthology assignments when two proteins had an E-value $\leq 10-5$ with $\geq 80 \%$ similarity over $\geq 70 \%$ of the gene and a Jaccard coefficient cutoff of 0.6.

\section{Availability of supporting data}

The T. parva schizont-stage RNA-seq data is available in the NCBI Sequence Read Archive (BioSample accession SAMN03981746).

\section{Additional files}

\section{Additional file 1: Supplemental figures with information on motif composition and distribution around transcription start and termination sites, UTRs and introns. (PDF 9.92 MB) \\ Additional file 2: Supplemental tables with information on motif composition and distribution relative to genes and gene ontology. (PDF 4.11 MB)}

\section{Abbreviations \\ PAS: polyadenylation signal; SVSP: subtelomere-encoded variable secreted protein; TpHN: T. parva host nucleus; TSS: transcription start site; TTS: transcription termination site; UORF: untranslated open reading frame; UTR: untranslated region.}

Competing interests

The authors declare they have no competing interests.

\section{Authors' contributions}

JCS and KT conceived the project and devised computational approaches, RP cultured the T. parva-infected lymphocytes and generated the RNA sample, KT performed the analyses, and all authors wrote and approved the manuscript.

\section{Acknowledgements}

This work was supported in part by the United States Department of Agriculture, through Scientific Cooperative Agreement \#58-5348-4-013 with JCS. 


\section{Author details}

Institute for Genome Sciences and Department of Microbiology and Immunology, University of Maryland School of Medicine, Baltimore, MD, USA. ${ }^{2}$ International Livestock Research Institute (ILRI), Nairobi, Kenya.

\section{Received: 17 September 2015 Accepted: 8 February 2016} Published online: 20 February 2016

\section{References}

1. Vollmer D. Enhancing the Effectiveness of Sustainability Partnerships Summary of a Workshop. 500 Fifth Street, N.W., Washington DC 20001: The National Academies Press; 2009.

2. Tretina K, Gotia HW, Mann DJ, Silva JC. Theileria-transformed bovine leukocytes have cancer hallmarks. Trends Parasitol. 2015;31(7):8.

3. De Goeyse I, Jansen F, Madder M, Hayashida K, Berkvens D, Dobbelaere D, et al. Transfection of live, tick derived sporozoites of the protozoan Apicomplexan parasite Theileria parva. Vet Parasitol. 2015;208(3-4):238-41.

4. Le Roch KG, Chung DW, Ponts N. Genomics and integrated systems biology in Plasmodium falciparum: a path to malaria control and eradication. Parasite Immunol. 2012;34(2-3):50-60.

5. Juven-Gershon T, Kadonaga JT. Regulation of gene expression via the core promoter and the basal transcriptional machinery. Dev Biol. 2010;339(2):225-9.

6. Sandelin A, Carninci P, Lenhard B, Ponjavic J, Hayashizaki Y, Hume DA. Mammalian RNA polymerase II core promoters: insights from genome-wide studies. Nat Rev Genet. 2007;8(6):424-36.

7. Balaji S, Babu MM, lyer LM, Aravind L. Discovery of the principal specific transcription factors of Apicomplexa and their implication for the evolution of the AP2-integrase DNA binding domains. Nucleic Acids Res. 2005;33(13): 3994-4006.

8. Essien K, Stoeckert Jr CJ. Conservation and divergence of known apicomplexan transcriptional regulons. BMC Genomics. 2010;11:147.

9. Oberstaller J, Pumpalova Y, Schieler A, Llinas M, Kissinger JC. The Cryptosporidium parvum ApiAP2 gene family: insights into the evolution of apicomplexan AP2 regulatory systems. Nucleic Acids Res. 2014;42(13):8271-84.

10. Campbell TL, De Silva EK, Olszewski KL, Elemento O, Llinas M. Identification and genome-wide prediction of DNA binding specificities for the ApiAP2 family of regulators from the malaria parasite. PLoS Pathog. 2010;6(10):e1001165.

11. Guo X, Silva JC. Properties of non-coding DNA and identification of putative cis-regulatory elements in Theileria parva. BMC Genomics. 2008;9:582.

12. Szafranski K, Lehmann R, Parra G, Guigo R, Glockner G. Gene organization features in A/T-rich organisms. J Mol Evol. 2005;60(1):90-8.

13. Russell K, Hasenkamp S, Emes R, Horrocks P. Analysis of the spatial and temporal arrangement of transcripts over intergenic regions in the human malarial parasite Plasmodium falciparum. BMC Genomics. 2013;14:267.

14. Hermsen R, ten Wolde PR, Teichmann S. Chance and necessity in chromosomal gene distributions. Trends Genet. 2008;24(5):216-9.

15. Ho MR, Tsai KW, Lin WC. A unified framework of overlapping genes: towards the origination and endogenic regulation. Genomics. 2012;100(4):231-9.

16. Gardner MJ, Bishop R, Shah T, de Villiers EP, Carlton JM, Hall N, et al. Genome sequence of Theileria parva, a bovine pathogen that transforms lymphocytes. Science. 2005;309(5731):134-7.

17. Pain A, Renauld H, Berriman M, Murphy L, Yeats CA, Weir W, et al. Genome of the host-cell transforming parasite Theileria annulata compared with T. parva. Science. 2005;309(5731):131-3.

18. Kappmeyer LS, Thiagarajan M, Herndon DR, Ramsay JD, Caler E, Djikeng A, et al. Comparative genomic analysis and phylogenetic position of Theileria equi. BMC Genomics. 2012;13:603.

19. Hayashida K, Hara Y, Abe T, Yamasaki C, Toyoda A, Kosuge T, et al. Comparative genome analysis of three eukaryotic parasites with differing abilities to transform leukocytes reveals key mediators of Theileria-induced leukocyte transformation. mBio. 2012;3(5):e00204-00212.

20. Imamura H, Persampieri JH, Chuang JH. Sequences conserved by selection across mouse and human malaria species. BMC Genomics. 2007:8:372.

21. Mullapudi N, Joseph SJ, Kissinger JC. Identification and functional characterization of cis-regulatory elements in the apicomplexan parasite Toxoplasma gondii. Genome Biol. 2009;10(4):34.
22. Yamagishi J, Wakaguri H, Yokoyama N, Yamashita R, Suzuki Y, Xuan X, et al. The Babesia bovis gene and promoter model: an update from full-length EST analysis. BMC Genomics. 2014;15:678.

23. Pieszko M, Weir W, Goodhead I, Kinnaird J, Shiels B. ApiAP2 factors as candidate regulators of stochastic commitment to Merozoite production in Theileria annulata. PLoS Negl Trop Dis. 2015;9(8):e0003933.

24. Zabel U, Schreck R, Baeuerle PA. DNA binding of purified transcription factor NF-kappa B. Affinity, specificity, Zn2+ dependence, and differential half-site recognition. J Biol Chem. 1991;266(1):252-60.

25. Pelechano V, Steinmetz LM. Gene regulation by antisense transcription. Nat Rev Genet. 2013;14(12):880-93.

26. Richard P, Manley JL. Transcription termination by nuclear RNA polymerases. Genes Dev. 2009:23(11):1247-69.

27. Kuehner JN, Pearson EL, Moore C. Unravelling the means to an end: RNA polymerase II transcription termination. Nat Rev Mol Cell Biol. 2011;12(5):283-94.

28. Mignone F, Gissi C, Liuni S, Pesole G. Untranslated regions of mRNAs. Genome Biol. 2002;3(3):REVIEWS0004

29. Jia J, Yao P, Arif A, Fox PL. Regulation and dysregulation of 3'UTR-mediated translational control. Curr Opin Genet Dev. 2013;23(1):29-34.

30. Beaudoing E, Freier S, Wyatt JR, Claverie JM, Gautheret D. Patterns of variant polyadenylation signal usage in human genes. Genome Res. 2000;10(7):1001-10.

31. Wilkie GS, Dickson KS, Gray NK. Regulation of mRNA translation by 5'- and 3'-UTR-binding factors. Trends Biochem Sci. 2003;28(4):182-8.

32. Babendure JR, Babendure JL, Ding JH, Tsien RY. Control of mammalian translation by mRNA structure near caps. RNA. 2006;12(5):851-61.

33. Hayden CA, Jorgensen RA. Identification of novel conserved peptide UORF homology groups in Arabidopsis and rice reveals ancient eukaryotic origin of select groups and preferential association with transcription factorencoding genes. BMC Biol. 2007:5:32.

34. Bunnik EM, Chung DW, Hamilton M, Ponts N, Saraf A, Prudhomme J, et al. Polysome profiling reveals translational control of gene expression in the human malaria parasite Plasmodium falciparum. Genome Biol. 2013;14(11):R128.

35. Yewdell JW, Anton LC, Bennink JR. Defective ribosomal products (DRiPs): a major source of antigenic peptides for MHC class I molecules? J Immunol. 1996;157(5):1823-6.

36. Crampton N, Bonass WA, Kirkham J, Rivetti C, Thomson NH. Collision events between RNA polymerases in convergent transcription studied by atomic force microscopy. Nucleic Acids Res. 2006;34(19):5416-25.

37. Hobson DJ, Wei W, Steinmetz LM, Svejstrup JQ. RNA polymerase II collision interrupts convergent transcription. Mol Cell. 2012;48(3):365-74.

38. Dahary D, Elroy-Stein O, Sorek R. Naturally occurring antisense: transcriptional leakage or real overlap? Genome Res. 2005;15(3):364-8.

39. Siegel TN, Hon CC, Zhang Q, Lopez-Rubio JJ, Scheidig-Benatar C, Martins RM, et al. Strand-specific RNA-Seq reveals widespread and developmentally regulated transcription of natural antisense transcripts in Plasmodium falciparum. BMC Genomics. 2014;15:150

40. Bright AT, Winzeler EA. Noncoding RNA, antigenic variation, and the virulence genes of Plasmodium falciparum. BMC Biol. 2011;9:50

41. Templeton TJ, Iyer LM, Anantharaman V, Enomoto S, Abrahante JE, Subramanian GM, et al. Comparative analysis of apicomplexa and genomic diversity in eukaryotes. Genome Res. 2004;14(9):1686-95.

42. Barry JD, Ginger ML, Burton P, McCulloch R. Why are parasite contingency genes often associated with telomeres? Int J Parasitol. 2003;33(1):29-45.

43. Schmuckli-Maurer J, Casanova C, Schmied S, Affentranger S, Parvanova I, Kang'a S, et al. Expression analysis of the Theileria parva subtelomereencoded variable secreted protein gene family. PLoS One. 2009;4(3):e4839.

44. Shiels BR, McKellar S, Katzer F, Lyons K, Kinnaird J, Ward C, et al. A Theileria annulata DNA binding protein localized to the host cell nucleus alters the phenotype of a bovine macrophage cell line. Eukaryot Cell. 2004;3(2):495-505.

45. Silva JC, Egan A, Arze C, Spouge JL, Harris DG. A new method for estimating species age supports the coexistence of malaria parasites and their Mammalian hosts. Mol Biol Evol. 2015;32(5):1354-64.

46. Young RS, Hayashizaki Y, Andersson R, Sandelin A, Kawaji H, Itoh M, et al. The frequent evolutionary birth and death of functional promoters in mouse and human. Genome Res. 2015;25(10):11.

47. Bailey TL, Boden M, Buske FA, Frith M, Grant CE, Clementi L, et al. MEME SUITE: tools for motif discovery and searching. Nucleic Acids Res. 2009;37(Web Server issue):W202-208.

48. Grant CE, Bailey TL, Noble WS. FIMO: scanning for occurrences of a given motif. Bioinformatics. 2011;27(7):1017-8. 
49. Mahony S, Benos PV. STAMP: a web tool for exploring DNA-binding motif similarities. Nucleic Acids Res. 2007;35(Web Server issue):W253-258.

50. Portales-Casamar E, Thongjuea S, Kwon AT, Arenillas D, Zhao X, Valen E, et al. JASPAR 2010: the greatly expanded open-access database of transcription factor binding profiles. Nucleic Acids Res. 2010;38(Database issue):D105-110.

51. Matys V, Fricke E, Geffers R, Gossling E, Haubrock M, Hehl R, et al. TRANSFAC: transcriptional regulation, from patterns to profiles. Nucleic Acids Res. 2003;31(1):374-8.

52. Down TA, Bergman CM, Su J, Hubbard TJ. Large-scale discovery of promoter motifs in Drosophila melanogaster. PLoS Comput Biol. 2007;3(1):e7.

53. Gonzalez C. Drosophila melanogaster: a model and a tool to investigate malignancy and identify new therapeutics. Nat Rev Cancer. 2013;13(3):172-83.

54. Yilmaz A, Mejia-Guerra MK, Kurz K, Liang X, Welch L, Grotewold E. AGRIS: the Arabidopsis Gene Regulatory Information Server, an update. Nucleic Acids Res. 2011;39(Database issue):D1118-1122.

55. Bulow L, Engelmann S, Schindler M, Hehl R. AthaMap, integrating transcriptional and post-transcriptional data. Nucleic Acids Res. 2009, 37(Database issue):D983-986.

56. Higo $\mathrm{K}$, Ugawa $\mathrm{Y}$, Iwamoto M, Korenaga T. Plant cis-acting regulatory DNA elements (PLACE) database: 1999. Nucleic Acids Res. 1999;27(1):297-300.

57. Hume MA, Barrera LA, Gisselbrecht SS, Bulyk ML. UniPROBE, update 2015: new tools and content for the online database of protein-binding microarray data on protein-DNA interactions. Nucleic Acids Res. 2015;43(Database issue):D117-122.

58. Maclsaac KD, Wang T, Gordon DB, Gifford DK, Stormo GD, Fraenkel E. An improved map of conserved regulatory sites for Saccharomyces cerevisiae. BMC Bioinformatics. 2006;7:113.

59. Harbison CT, Gordon DB, Lee TI, Rinaldi NJ, Macisaac KD, Danford TW, et al. Transcriptional regulatory code of a eukaryotic genome. Nature. 2004;431 (7004): 99-104.

60. Cipriano MJ, Novichkov PN, Kazakov AE, Rodionov DA, Arkin AP, Gelfand MS, et al. RegTransBase-a database of regulatory sequences and interactions based on literature: a resource for investigating transcriptional regulation in prokaryotes. BMC Genomics. 2013;14:213.

61. Robison K, McGuire AM, Church GM. A comprehensive library of DNA-binding site matrices for 55 proteins applied to the complete Escherichia coli K-12 genome. J Mol Biol. 1998:284(2):241-54.

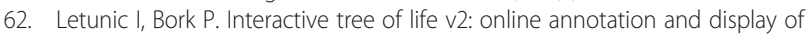
phylogenetic trees made easy. Nucleic Acids Res. 2011;39(Web Server issue): W475-478.

\section{Submit your next manuscript to BioMed Central and we will help you at every step:}

- We accept pre-submission inquiries

- Our selector tool helps you to find the most relevant journal

- We provide round the clock customer support

- Convenient online submission

- Thorough peer review

- Inclusion in PubMed and all major indexing services

- Maximum visibility for your research

Submit your manuscript at www.biomedcentral.com/submit 